\title{
Analisis De La Percepcion De Alumnos Y Egresados Para Determinar Y Promover EI Sentido De Pertenencia Y La Filantropia En Universidades
}

\author{
María Guadalupe Huesca Ramírez, Dra. \\ Marivel Lumbreras Guzmán, Dra. \\ Irma Alejandra Hernández Vicente, Dra. \\ Gaspar Eduardo Fuentes Garza, MBA
}

Universidad Popular Autónoma del Estado de Puebla, Mexico

Doi: 10.19044/esj.2017.v13n35p206 URL:http://dx.doi.org/10.19044/esj.2017.v13n35p206

\begin{abstract}
The objective of this article is to define what are the factors that prevent achieving a high sense of belonging in current students and graduates of a private university. The sense of belonging impacts on the level of participation of the activities triggered by the university as well as philanthropic strategies that seek to support those who need it most. To carry out this study, group sessions were held with graduates and current students. The latter were also given a survey to know their current perception of belonging and desire to participate in what promotes their university for which the result is that $94 \%$ of students would support philanthropic initiatives. On the other hand, the graduates comment that there is a lack of better communication to know what is happening in their alma mater and also to design linking models to strengthen current ties.
\end{abstract}

Keywords: Membership, philanthropy

\section{Resumen}

El objetivo de este artículo es definir cuáles son los factores que impiden lograr un alto sentido de pertenencia en los alumnos actuales y egresados de una universidad privada. El sentido de pertenencia impacta en el nivel de participación de las actividades que detona la universidad así como también en estrategias de carácter filantrópico que buscan apoyar a quienes más lo necesitan. Para la realización de este estudio se llevaron a cabo sesiones grupales con egresados y alumnos actuales. A estos últimos también se les aplicó una encuesta para conocer su perceptación actual de pertenencia y deseo de participación en lo que promueva su universidad para 
lo cual el resultado obtenido es que un $94 \%$ de los alumnos sí apoyaría iniciativas filantrópicas. Por otra parte los egresados comentan que falta una mejor comunicación para conocer lo que ocurre en su alma mater y también el diseñar modelos de vinculación para fortalecer los lazos actuales.

Palabras clave: Pertenencia, filantropía

\section{Introducción}

En un país donde los indicadores de filantropía retroceden en lugar de avanzar (World Giving Index, 2016) es importante implementar estrategias que la fomenten, pero sobre todo que la actitud de servicio y ayuda se vuelva un estilo de vida para los seres humanos. Sin duda, el efecto que se produce en una entidad donde la filantropía forma parte de la formación de las personas, se ve reflejada en el desarrollo de su comunidad. Existen una gama de ejemplos que se pueden tomar en cuenta para garantizar que esta actividad puede generar resultados valiosos, si se aplica correctamente.

La pertenencia es sin duda un potencializador de cualquier iniciativa a favor de un bien mayor, y la educación sin duda, no es la excepción, ya que es uno de los factores primordiales para alcanzar el desarrollo en un país. En México, el tema de la educación ocupa uno de los primeros lugares en cuanto a apoyo filantrópico, sin embargo, es mínimo el apoyo que se recibe por parte de las empresas y/o personas que existen en México.

\section{Marco Teórico}

El origen de las instituciones universitarias, representa parte fundamental en el desarrollo académico de todo el mundo, sin embargo al ser éste un tema tan extenso, para efectos de esta investigación, se abordará a grandes rasgos el origen de la educación superior en México, así como el de algunas instituciones universitarias.

(Torres, 1997: parr. 6) explica que la Educación privada en México se inicia desde tiempos de la colonia cuando los llamados preceptores se ocupaban de la educación individual de niños, niñas y jóvenes.

Las universidades privadas de México con prestigio por sus profesores, programas y egresados de acuerdo con el ranking 2015, ofrecen esquemas de apoyo y financiamiento de la educación mediante becas y préstamos propios o bancarios para los alumnos que deseen cursar la licenciatura. Los costos de la educación privada en nivel superior llegan a situarse hasta en 200 mil pesos anuales, dependiendo de la carrera y la institución. No obstante, las universidades particulares se han dado a la tarea de ofrecer becas y préstamos para financiar desde el 10 hasta el 100 por ciento de las carreras. (Martínez, 2015: parr. 3). 
El apoyo económico (becas) que ofrecen las universidades privadas a algunos alumnos, son recursos monetarios que de alguna manera la institución tiene que recuperar ese capital invertido en sus alumnos, por lo tanto, debido a que la mayoría de las universidades del sector privado no cuentan con el apoyo económico por parte del gobierno, dichas instituciones día con día están en busca de atraer recursos económicos que les permita seguir ofreciendo una formación académica a sus estudiantes y sobre todo financiando a aquellos que no cuentan con la solvencia económica para cursar sus estudios universitarios en una escuela particular.

Cuando una universidad de la iniciativa privada recibe ingresos extras mediante acciones filantrópicas, ésta es capaz de ofrecer una formación académica sobresaliente puesto que cuenta con los recursos necesarios para hacerlo, de igual manera puede fortalecer su infraestructura y puede ser capaz de llegar a convertirse en una organización donde laboralmente sus colaboradores puedan continuar con su desarrollo personal y profesional.

Carrillo \& Vargas (2008: p.6) mencionan que la filantropía permite que la sociedad civil crezca y se democratice, puede ser un instrumento estratégico de cambio social si se vincula con las causas, los problemas, los actores, las regiones y los enfoques que promueven este cambio y que las acciones filantrópicas comienzan por una actitud, una preocupación y un valor de solidaridad.

En México existen diferentes áreas en las cuales las organizaciones aportan sus donaciones, de acuerdo con Carrillo, (2009: p. 30) las principales áreas de inversión social por parte de las empresas se pueden observar en la siguiente tabla:

Tabla 1. Distribución de la inversión social por áreas en México.

\begin{tabular}{|c|c|}
\hline Área de inversión social: & Porcentaje de apoyo \\
\hline Educación & $73 \%$ \\
\hline Medio ambiente & $58 \%$ \\
\hline Desarrollo comunitario & $58 \%$ \\
\hline Niños & $58 \%$ \\
\hline Discapacitados & $43 \%$ \\
\hline
\end{tabular}

Tomando como base lo señalado por Carrillo (2009: p. 30.) donde manifiesta que la educación ocupa el primer lugar en las áreas de apoyo, las universidades de la iniciativa privada pueden recurrir a diferentes fórmulas para atraer recursos económicos y lograr que las personas directamente involucradas que se sientan atraídas por las actividades que implementan en la institución y que de esta manera el área educativa continúe siendo una de las áreas con mayor apoyo por parte de las empresas dedicadas a ayudar a otras organizaciones. 
Indudablemente las fórmulas que desarrolle cada universidad, para la captación de recursos monetarios, deben estar dirigidas en primera instancia a sus alumnos y egresados, por lo tanto, la institución debe diseñar estrategias de pertenencia e identidad universitaria que haga que los alumnos y exalumnos se sientan comprometidos con las actividades filantrópicas impulsadas por la propia universidad.

Cortes (2011: p.79) menciona que la identidad universitaria se encuentra inscrita en la manera en como la institución se define a sí misma y actúa en un contexto determinado; se encuentra también en sus marcos valorativos, en sus repertorios culturales y en sus formas de organización; en sus antecedentes históricos (...) los proyectos de internacionalización que sirven de guía a sus tareas, y, por supuesto, en su responsabilidad social.

Aunado a lo citado por Cortes, (2011: p. 79), Valdez, (2013: parr. 1) señala que toda organización universitaria debe contar con un programa que ayude a los integrantes de la misma a sentirse ligados a la organización y que refuerce la identidad que toda la comunidad universitaria, al respecto, Rodas ( 2010: p. 3) menciona que una persona con sentido de pertenencia puede tener un mayor grado de compromiso, afecto y motivación, lo cual incide en la realización de un trabajo altamente efectivo y eficiente que al sumarse con el trabajo del equipo le genera al sistema: desarrollo, calidad, productividad e innovación, lo que repercute en el fortalecimiento de la imagen institucional dando inicio al sentido de pertenencia, sustentando lo expuesto por Rodas ( 2010).

Fomentar acciones filantrópicas trae consigo múltiples beneficios para la sociedad que integra una comunidad, la Fundación Vamos a Dar (2016) da a conocer cinco beneficios que por los cuales es conveniente impulsar el trabajo social.

- Las Organizaciones de la Sociedad Civil (OSC) contribuyen a impulsar la economía nacional.

- $\quad$ Las OSC mejoran la percepción pública de las instituciones.

- Las alianzas entre gobierno, empresas y OSC's estimulan la prosperidad de la sociedad.

- La cultura solidaria fortalece los tejidos sociales.

- Las actividades de la OSC promueven mecanismos que ayudan a satisfacer las necesidades de la sociedad cuando el esfuerzo y capacidad humana que demandan son muy grandes.

Es probable que no todos los alumnos y egresados se comprometan con las actividades filantrópicas de la universidad, ya que no todos desarrollan el mismo grado de pertenencia a la institución. La escasa participación puede ser provocada por diversos factores que impactan negativamente los planes y proyectos de la Institución, entre dichos factores destacan: 
- La apatía a las actividades sociales de la universidad.

- La falta de sentido de pertenencia a la institución.

- $\quad$ Desconocimiento del área filantrópica de la universidad.

- $\quad$ La falta de compromiso.

- $\quad$ La falta de recursos económicos.

\section{Metodología}

Esta investigación busca determinar los factores que promueven el sentido de pertenencia en alumnos actuales y egresados de instituciones de educación superior de caracter privado las cuales, permitan una mayor participación en las actividades organizadas por dichas escuelas así como en iniciativas filantrópicas y de procuración de fondos que permitan impulsar los proyectos que se gestan dentro de la institución.

El proceso de evaluación se realizó con una metodología descriptiva que ayudó a detallar cómo se presenta una situación determinada. Se emplearon dos tipos de investigación, por un lado se adoptó la cuantitativa para aplicar una encuesta de línea base que nos permitiera conocer el sentido de pertenencia y vinculación de los alumnos actuales. Por otro lado se empleó la investigación cualitativa que implicó recolectar la opinión de alumnos actuales y egresados a través de grupos de enfoque para entender las causas de la baja participación las actividades promovidas por su universi dad así como en la vinculación filantrópica.

\section{Detalle de la Implementación}

Para obtener la información necesaria que permita conocer la percepción actual de los alumnos actuales y egresados y así poder crear estrategias para promover el sentido de pertenencia y una mayor participación filantrópica en ambas poblaciones, se formaron dos grupos con los cuales se trabajó para obtener dichos datos.

Grupo 1 - Exalumnos: conformado por egresados de la universidad que tienen más de 5 años de haber finalizado su nivel de licenciatura.

Grupo 2 - Alumnos actuales: alumnos que actualmente están cursando sus estudios de profesional en instituciones privadas.

Para el grupo 1, la suma de los egresados, es de 120 mil, de los cuales, sólo el 5\% tiene una participación activa, esto quiere decir que sólo 6 mil egresados participan activamente ya sea donando ó teniendo algún tipo de vinculación con su alma mater.

En el caso del grupo 2, la suma de los alumnos actualmente inscritos en carreras profesionales es de 15 mil estudiantes.

Para el Grupo 1 (exalumnos) se definió un esquema de grupos de enfoque (focus groups), Lo anterior se determinó debido a la poca participación por parte de los egresados y también porque no se tiene 
información actualizada de contacto para hacerles llegar un vínculo para contestar la encuesta. Se llevaron a cabo 15 sesiones de grupos de enfoque noc 20 participantes cada una de ellas con egresados de distintas carreras y años de graduados.

Para el Grupo 2 (alumnos actualmente inscritos) se definieron dos esquemas: La aplicación de 450 encuestas en formato electrónio, si buscamos un nivel de confianza del $95 \%$ se ocuparían al menos 379 encuestas, sin embargo, para esta investigación se aplicaron 450.

También para este grupo se programaron 10 sesiones de grupos de enfoque con una participación de 20 alumnos cada una de ellas.

La encuesta, nos permitirá tener una línea base sobre el nivel de pertenencia que tienen los alumnos y también su opinión sobre las iniciativas que se detonan en la universidad así como la comunicación correspondiente.

Esta encuesta fue de diseño propio, basada en instrumentos de diaganóstico sugeridos por el Council for advancement and support of education (Case, 2017) y reúne los elemantos básicos que se desean conocer de los alumnos actuales

Por su parte, los grupos de enfoque, son los que nos ayudarán a tener una mayor sensibilidad de lo que piensan y sienten los alumnos y egresados y también serán clave para generar estrategias bien enfocadas que permitan lograr un mayor sentido de pertenencia y compromiso con las causas de la universidad.

\section{Resultados}

\section{Sesiones de enfoque:}

El desarrollo de los grupos de enfoque se llevó a cabo en sesiones, atendiendo los factores básicos de estados de grupos para este tipo de instrumento, los cuales son la pertinencia y la consistencia que recomiendan al menos dos grupos de enfoque por cada característica variable, una muestra es que hubo sesiones con egresados de 1-5 años, 5-10 años y 10-15 años de graduados así como también se llevaron a cabo sesiones con alumnos actuales de todas las carreras y todos los semestres.

En la siguiente tabla se presentan las preguntas detonantes que se hicieron en la sesión grupal tanto con egresados así como con alumnos y un resumen de las conclusiones generadas para cada uno de estos cuestionamientos. 
Tabla 2. Resumen de los resultados generados por los grupos de enfoque a alumnos y egresados.

\begin{tabular}{|c|c|}
\hline Preguntas & Respuestas \\
\hline $\begin{array}{l}\text { Porque en nuestro país hay una baja } \\
\text { participación en actividad de } \\
\text { filantropía. }\end{array}$ & $\begin{array}{l}\text { No se promueve el espíritu filantrópico desde la niñez. } \\
\text { No son claras las causas que se apoyan ni la aplicación de los } \\
\text { recursos. }\end{array}$ \\
\hline $\begin{array}{c}\text { ¿A qué se debe la baja participación } \\
\text { de los en actividades diversas que } \\
\text { organiza la universidad? }\end{array}$ & $\begin{array}{l}\text { No se enteran de los eventos que ocurren. } \\
\text { Sólo se les busca para pedirles algo }\end{array}$ \\
\hline $\begin{array}{l}\text { ¿Por qué los egresados no participan } \\
\text { en las iniciativas filantrópicas que se } \\
\text { hacen en la universidad? }\end{array}$ & $\begin{array}{l}\text { La institución ya tiene recursos gracias a sus colegiaturas. } \\
\text { Quienes tuvieron apoyo educativo perciben que ya pagaron } \\
\text { sus créditos con intereses. }\end{array}$ \\
\hline $\begin{array}{l}\text { ¿Qué acciones se tienen que hacer } \\
\text { para promover el sentido de } \\
\text { pertenencia en los alumnos actuales? }\end{array}$ & $\begin{array}{c}\text { Promover una mayor participación de los alumnos en los } \\
\text { planes y proyectos de la institución. } \\
\text { Que los profesores promuevan más los logros de la } \\
\text { institución. }\end{array}$ \\
\hline $\begin{array}{l}\text { ¿Qué acciones hay que realizar para } \\
\text { que los egresados participen en las } \\
\text { actividades de su Universidad? }\end{array}$ & $\begin{array}{l}\text { Invitarlos a eventos y conferencias de primer nivel que se } \\
\text { lleven a cabo. } \\
\text { Mejorar el proceso de comunicación con los egresados. }\end{array}$ \\
\hline $\begin{array}{c}\text { ¿Qué se tendría que hacer para } \\
\text { promover el sentido de pertenencia? }\end{array}$ & $\begin{array}{l}\text { Que los alumnos participen en la toma de decisiones. } \\
\text { Crear "rituales" para la admisión, la entrega de becas y } \\
\text { graduaciones. }\end{array}$ \\
\hline $\begin{array}{l}\text { ¿Qué acciones se tienen que hacer } \\
\text { para promover el sentido de } \\
\text { pertenencia y el acercamiento con los } \\
\text { alumnos egresados? }\end{array}$ & $\begin{array}{l}\text { Implementar programas de mentoreo donde un egresado } \\
\text { "adopte" a un alumno. } \\
\text { Esquemas de financiamiento menos agresivos para los } \\
\text { alumnos que tuvieron crédito educativo. }\end{array}$ \\
\hline $\begin{array}{l}\text { ¿Qué esfuerzos de filantropía serían } \\
\text { los que más sentirías afines para } \\
\text { apoyar? }\end{array}$ & $\begin{array}{c}\text { Becas académicas para alumnos. } \\
\text { Becas de sostenimiento. } \\
\text { Apoyos para que los alumnos realicen su estancia en el } \\
\text { extranjero. }\end{array}$ \\
\hline
\end{tabular}

\section{Encuesta a alumnos actuales:}

\section{A continuación se presentan las preguntas realizadas en las encuesta} que se aplicó a los alumnos actuales y los resultados obtenidos en cada una de ellas:

\begin{tabular}{|c|c|c|}
\hline Pregunta & Texto & Descripción \\
\hline 1 & $\begin{array}{c}\text { Edad de los alumnos encuestados } \\
\text { dentro de la universidad en la cual } \\
\text { se está llevando a cabo esta } \\
\text { investigación }\end{array}$ & $\begin{array}{c}\text { El 63\% de la población encuestada mantiene } \\
\text { una edad cuyo rango es de los 21 a los 25 años } \\
\text { de edad. El 36\% lo ocupa una población con } \\
\text { una edad que oscila entre 18 y 20 años. } \\
\text { Finalmente, solo el } 1 \% \text { de la población } \\
\text { encuestada. }\end{array}$ \\
\hline 2 & Género de los encuestados & $\begin{array}{c}\text { El 60\% de los alumnos encuestados } \\
\text { corresponden al género femenino, mientras que } \\
\text { el 40\% restante son del género masculino. }\end{array}$ \\
\hline 3 & Lugar de origen de los encuestados & $\begin{array}{c}\text { El 67\% de la población encuestada es de origen } \\
\text { local (Monterrey y área metropolitana) y el } \\
33 \% \text { es foránea. }\end{array}$ \\
\hline 4 & Carrera profesional de los \\
encuestados & $\begin{array}{c}\text { Del total de los encuestados, se tiene que la } \\
\text { mayoría de los encuestados cursa la carrera de } \\
\text { Ingeniero Químico Administrador (9.36\%), } \\
\text { enseguida le sigue la de Ingeniero Biomédico } \\
(6.81 \%) \text { y, en tercer lugar, la de Ingeniero }\end{array}$ \\
\hline
\end{tabular}




\begin{tabular}{|c|c|c|}
\hline & & Industrial y de Sistemas (6.38 \%). \\
\hline 5 & Semestre en curso & $\begin{array}{l}\text { Del total de alumnos encuestados se tiene que: } \\
\text { el } 35 \% \text { de la población se encuentra cursando } \\
\text { el } 6 .^{\circ} \text { semestre; el } 18 \% \text { cursa el } 5 .^{\circ} \text { semestre; el } \\
15 \% \text { de los encuestados, el } 7 .^{\circ} \text { semestre; el } 9 \% \\
\text { está en el } 4 .^{\circ} \text { semestre; también otro } 9 \% \text { de la } \\
\text { población cursa el } 9 .^{\circ} \text { semestre; un } 7 \% \text { cursa el } \\
10 .^{\circ} \text { semestre o más; y otro } 7 \% \text { se encuentra en } \\
\text { el } 8 .^{\circ} \text { semestre. }\end{array}$ \\
\hline 6 & Ser parte de la universidad & $\begin{array}{l}\text { El } 33 \% \text { de la población encuestada se siente } \\
\text { totalmente parte de la universidad, un } 40 \% \\
\text { manifiesta no sentirse totalmente parte de la } \\
\text { institución, } 16 \% \text { de los estudiantes se sienten } \\
\text { indecisos en ese sentir, } 8 \% \text { no se siente del todo } \\
\text { parte de la universidad y un } 3 \% \text { manifiesta no } \\
\text { sentirse totalmente parte de la institución. }\end{array}$ \\
\hline 7 & Pertenencia a la universidad & $\begin{array}{c}\text { El } 63 \% \text { de los encuestados valora totalmente } \\
\text { formar parte de la universidad, el } 29 \% \text { valora } \\
\text { en menor grado formar parte de la universidad, } \\
\text { el } 5 \% \text { se siente indeciso al responder esta } \\
\text { pregunta y un } 3 \% \text { no valora ser parte de la } \\
\text { institución. }\end{array}$ \\
\hline 8 & $\begin{array}{c}\text { Comunicación entre la universidad } \\
\text { y comunidad estudiantil }\end{array}$ & $\begin{array}{c}\text { El } 34 \% \text { de los encuestados se manifestó de } \\
\text { acuerdo con la comunicación que hay entre la } \\
\text { universidad y ellos, el } 32 \% \text { está totalmente de } \\
\text { acuerdo, el } 25 \% \text { respondió estar indeciso, el } \\
7 \% \text { está en desacuerdo y un } 2 \% \text { está totalmente } \\
\text { en desacuerdo con la comunicación que se } \\
\text { genera entre la institución y la comunidad } \\
\text { estudiantil. }\end{array}$ \\
\hline 9 & $\begin{array}{c}\text { Comunicación para la generación } \\
\text { de proyectos e iniciativas }\end{array}$ & $\begin{array}{l}\text { El } 37 \% \text { de los encuestados respondió que la } \\
\text { universidad escucha sus opiniones para la } \\
\text { generación de proyectos, el } 31 \% \text { se siente } \\
\text { indeciso en esta cuestión, el } 15 \% \text { respondió } \\
\text { estar totalmente de acuerdo con que la } \\
\text { universidad escucha sus opiniones, el } 13 \% \text { se } \\
\text { siente en desacuerdo en este mismo aspecto y } \\
\text { un } 4 \% \text { está en total desacuerdo. }\end{array}$ \\
\hline 10 & Solidaridad con la universidad & $\begin{array}{l}\text { El } 94 \% \text { de la población mencionó que sí } \\
\text { apoyaría a la universidad en actividades } \\
\text { filantrópicas, mientras que solo un } 6 \% \text { expresó } \\
\text { lo contrario. }\end{array}$ \\
\hline 11 & Apoyo a iniciativa filantrópica & $\begin{array}{c}\text { El } 94 \% \text { de los alumnos encuestados sí apoyaría } \\
\text { alguna actividad filantrópica impulsada por la } \\
\text { universidad, mientras que el } 6 \% \text { se mostró } \\
\text { renuente a brindar este apoyo. }\end{array}$ \\
\hline
\end{tabular}

De los resultados generados, los siguientes puntos resumen algunos de los principales hallazgos:

- $\quad$ El 46\% de los alumnos considera que la universidad escucha sus opiniones para generar sus proyectos. Pero existe un 54\% que no está plenamente convencido de que se les escuche. 
- $\quad$ Un $\mathbf{7 7 \%}$ se siente parte de la universidad pero hay un $\mathbf{1 6 \%}$ que se siente indeciso al respecto y $\mathbf{1 7 \%}$ que no se siente identificado.

- $\quad$ El 94\% de los alumnos apoyarían iniciativas filantrópicas. Sólo un 6\% dijo que no lo haría.

- $\quad$ Del grupo de personas que estarían dispuestas a apoyar una inicitiva filantrópica, el 49\% apoyaría iniciativas de becas académicas, un 19\% para becas de sostenimiento (apoyo para alimentación, transporte, libros, etc.), un $14 \%$ apoyaría a equipos representativos y el $4 \%$ restante apoyaría otras iniciativas.

\section{Conclusion}

De acuerdo al análisis de la información generada por los grupos de enfoque así como en la encuesta, podemos concluir lo siguiente:

1. Hay desconocimiento de los programas filantrópicos que se detonan en la universidad.

2. Falta integrar la cultura filantrópica desde las bases de enseñanza de la población.

3. Se requiere mucha transparencia en la aplicación de los recursos obtenidos para que esto brinde tranquilidad a los donantes y la seguridad de que se aplican en forma adecuada.

4. Falta hacer partícipes a los alumnos de los procesos de toma de decisiones para que ellos se sientan dueños de los mismo y tengan mayor integración.

5. El tener un $23 \%$ del estudiantado con una sensación de falta de pertenencia es un reto importante ya que el objetivo es que todos se sientan muy orgullos de su institución.

6. El tener un $94 \%$ de alumnos dispuestos a apoyar la filantropía se debe aprovechar para potencializar el impacto de los proyectos.

Con la información anterior se pueden definir las siguientes líneas de acción que permitan promover la participación en actividades de la universidad así como la filantropía.

1. Generar estrategias de comunicación que permitan dar a conocer las actividades filantrópicas que se detonan en la universidad y el impacto que tienen en grupo al que se está apoyando.

2. Establecer programas de mentoreo en el que los egresados sean guías de los alumnos actuales en su estancia en la universidad así como para su integración a la vida profesional.

3. Diseñar esquemas de financiamiento educativos flexibles que permitan al egresado cumplir sus compromisos sin tanta presión sobre todo al inicio de su via profesional. 
4. Convertir al profesor en el embajador ante los alumnos tanto actuales como egresados ya que es una persona que tiene una gran ascendencia positiva y será más fácil que sea escuchado por ambos grupos.

\section{References:}

1. Council for advancement and support of education. (2017). Fundraising Fundamentals. Recuperado de: http://www.case.org/Publications_and_Products/Fundraising_Funda mentals_Intro/Fundraising_Fundamentals_section_1/Fundraising_Fu ndamentals_section_16.html

2. Carrillo, P. \& Vargas, S. (2009). Diagnóstico de la Filantropía Corporativa en México. Recuperado de: http://www.filantropia.itam.mx/docs/DiagnosticoFilCorp.pdf

3. Charities Aid Fundation. (2016) Caf World Giving Index 2016, The world's leading study of generosity. Recuperado de www.cafonline.org

4. Cortes, D. (2011). Aportes para el estudio de la identidad institucional universitaria. El caso de la UNAM. Perfiles Educativos

14 Recuperado

de http://www.redalyc.org/articulo.oa?id=13221258008

5. Forbes. (2015). Las empresas más benefactoras en México. Ciudad de México, México: Forbes. Recuperado de $\mathrm{http} / / / \mathrm{www}$.forbes.com.mx/las-empresas-mas-benefactoras-enmexico/\#gs.bA3_8fw

6. Fundación Vamos a Dar (2016). 5 razones para promover la filantropía en México. Recuperado de http://www.vamosadar.org.mx/noticias/?p=296

7. Garcés, R. y Díaz, A. (2015). ¿El lugar o el vínculo entre actores sociales? Lo comunitario en la gestión del desarrollo local. Retos de la Dirección, (1), 218. Recuperado de http://scielo.sld.cu/scielo.php?script=sci_arttext\&pid=S2306$91552015000100011 \& \operatorname{lng}=$ en\&tlng=en

8. Latin Trade. (2014). Filantropía en pensamiento y acción. 22(5), 88. Recuperado de http://latintrade.com/es/

9. Martinez, E. (2015, Mayo, 13) Educación superior privada, si está a tu alcance. El Financiero. Recuperado de: http://www.elfinanciero.com.mx/mis-finanzas/educacion-superiorprivada-si-esta-a-tu-alcance.html

10. Rodas, P. A., Ospina, L. M., \& Lanzas, A. M. (2010). Medición del Sentido de Pertenencia a partir de un Diseño Factorial con dos Factores. Scientia Et Technica, (44), 111. Recuperado de http://www.redalyc.org/articulo.oa?id=84917316020 
11. Torres, V. (1997) Educación Privada en México. Recuperado de http://biblioweb.tic.unam.mx/diccionario/htm/articulos/sec_20.htm

12. Valdez, A. (18 de febrero del 2013). Identidad Universitaria. La Gaceta de la Universidad de Guadalajara. Recuperado de http://gaceta.udg.mx/G_nota1.php?id=13427 